\title{
Identifying Atoms in High Resolution Transmission Electron Micrographs Using a Deep Convolutional Neural Net
}

\author{
Jakob Schiøtz ${ }^{1}$, Jacob Madsen ${ }^{1}$, Pei Liu ${ }^{2}$, Ole Winther ${ }^{3}$, Jens Kling ${ }^{2}$, Jakob Birkedal Wagner ${ }^{2}$ and \\ Thomas Willum Hansen ${ }^{2}$ \\ 1. Department of Physics, Technical University of Denmark, Kgs. Lyngby, Denmark. \\ 2. Center for Electron Nanoscopy, Technical University of Denmark, Kgs. Lyngby, Denmark. \\ 3. Department of Applied Mathematics and Computer Science, Technical University of Denmark, Kgs. \\ Lyngby, Denmark.
}

We have developed a deep-learning approach for recognizing local structures in high resolution transmission electron microscopy (HRTEM) images, which is stable to microscope parameters and noise. We train a deep convolutional neural network entirely from simulated HRTEM images, which is then able to reliably identify the positions of the atoms in experimental micrographs of single sheets of defected graphene, and the atomic columns in micrographs of metallic nanoparticles, even when the signal-to-noise ratio is low due to the desire to limit beam exposure.

We have applied the method to analyze surface diffusion on ceria supported gold nanoparticles, where we find that an oxygen atmosphere dramatically increase the diffusion (Figure 1) [1]. The neural net enables us to rapidly identify the presence or absence of individual columns of atoms in a time sequence of HRTEM micrographs.

While conventional image processing and peak finding algorithms can be used to pinpoint the position of the atoms (within the uncertainty due to imaging effects) [2], the fact that contrast varies from image to image, and sometimes even inverts within a single micrograph makes such methods difficult to use. The neural net acts as an advanced filter, giving a clean signal for peak detection (Figure 1).

While a single HRTEM image contains sufficient information for the neural net to identify the positions of the atoms, more information can be extracted from a focal series. We have demonstrated, albeit in simulations only, that a set of three images taken at different defocus is sufficient to identify chemical species and vacancies in single sheets of molybdenum disulfide (Figure 2), and to count the number of atoms in atomic columns in gold nanoparticles [1], which has until now only been possible with STEM.

In conclusion, we find that deep learning is a promising tool within electron microscopy, which may both accelerate analysis of large amounts of microscopy data and enable analyses that would not otherwise be possible, such as counting the height of atomic columns in HRTEM images.

References:

[1] J. Madsen et al, (to be published, https://arxiv.org/abs/1802.03008).

[2] J. Madsen et al, Adv. Struct. Chem. Imag. 3 (2017), p. 14. 

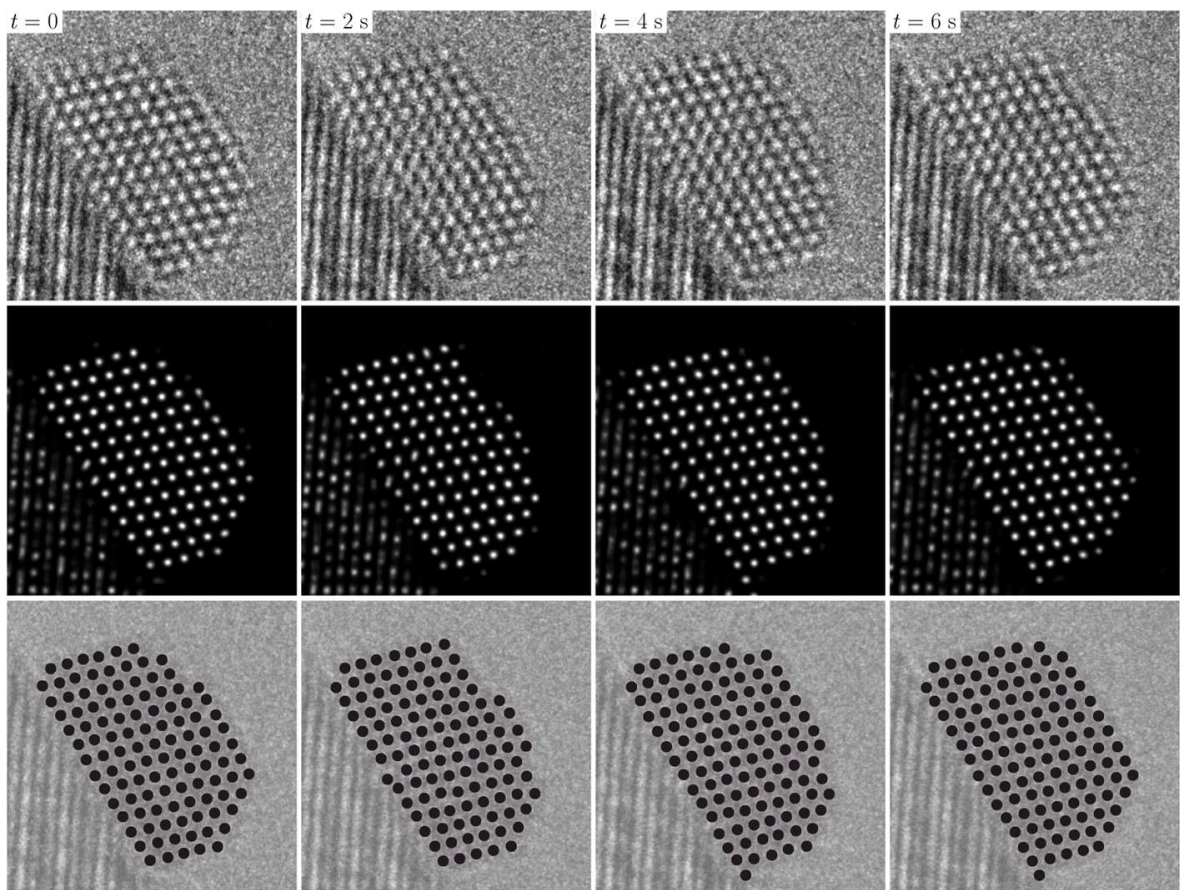

Figure 1. Top row: Experimental HRTEM image of gold on ceria in an oxygen atmosphere. Middle row: Output from the neural network given the images above. Bottom row: The atoms identified by the neural net are marked as dark circles overlaid on the original image. The experimental images were measured using a FEI Titan 80-300 Environmental TEM operated at $300 \mathrm{keV}$, the dose rate was $1.56 \times 10^{3} \mathrm{e}^{-} /\left(\AA^{2} \mathrm{~s}\right)$ and the $\mathrm{O}_{2}$ pressure was $4.5 \mathrm{~Pa}$. Reproduced from [1].
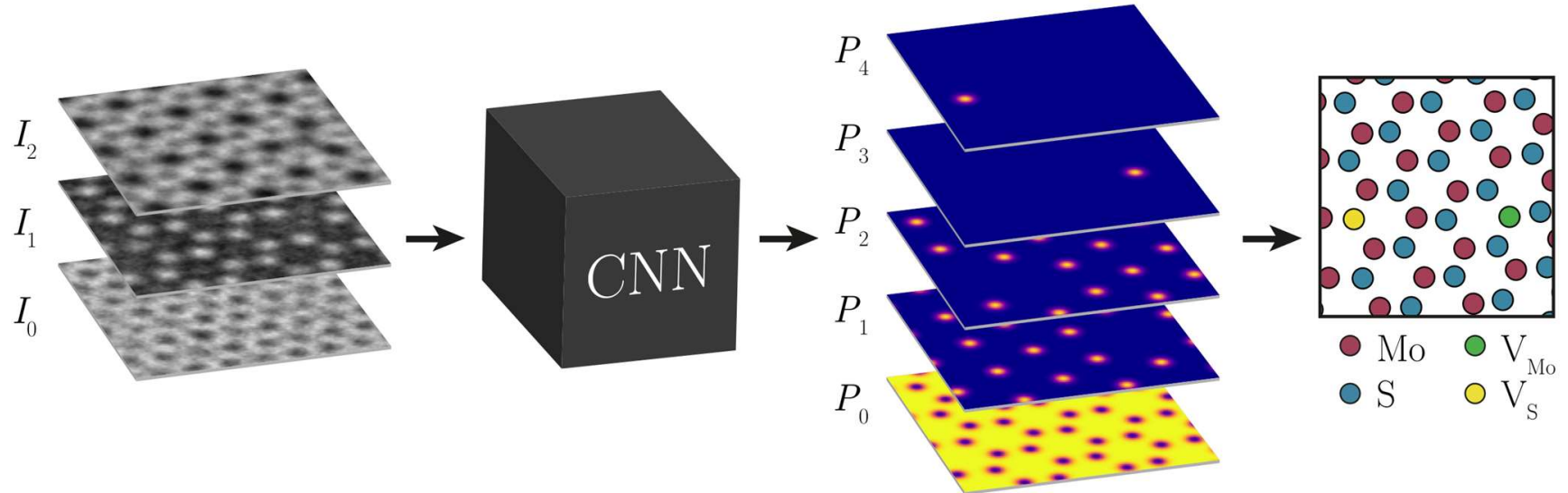

Figure 2. The neural net is fed three images of the same sample with different defocus. The output of the net is the probability of finding an "object" of a specific type at a given point. In this case, the set of possible objects are Mo atom, S atom, Mo vacancy, S vacancy and background. 